\title{
A no-go related prefrontal negativity larger to irrelevant stimuli that are difficult to suppress
}

\author{
Alice M Proverbio*1, Marzia Del Zotto ${ }^{1}$, Nicola Crotti ${ }^{1,2,3}$ and Alberto Zani ${ }^{3}$
}

\author{
Address: ${ }^{1}$ Dept. of Psychology, University of Milano-Bicocca, Milan, Italy, ${ }^{2}$ San Raffaele Hospital, HSR Monte Tabor Foundation, Milan, Italy and \\ ${ }^{3}$ Inst. of Bioimaging and Molecular Physiology, CNR, Milano-Segrate, Italy \\ Email: Alice M Proverbio* - mado.proverbio@unimib.it; Marzia Del Zotto - m.delzotto1@campus.unimib.it; \\ Nicola Crotti - n.crotti@campus.unimib.it; Alberto Zani - alberto.zani@ibfm.cnr.it \\ * Corresponding author
}

Published: 25 June 2009

Behavioral and Brain Functions 2009, 5:25 doi:10.1186/1744-908I-5-25
Received: 17 April 2009

Accepted: 25 June 2009

This article is available from: http://www.behavioralandbrainfunctions.com/content/5///25

(c) 2009 Proverbio et al; licensee BioMed Central Ltd.

This is an Open Access article distributed under the terms of the Creative Commons Attribution License (http://creativecommons.org/licenses/by/2.0), which permits unrestricted use, distribution, and reproduction in any medium, provided the original work is properly cited.

\begin{abstract}
Background: There is a wide debate in the literature about whether N2/P3 effects in no-go trials reflect the inhibition of an intended action, or the absence of a negative movement-related potential typical of go trials. The aim of this study was to provide an objective measure of the suppression of irrelevant information (in a conjoined selective visual attention task) under conditions that were perfectly comparable from the viewpoint of the motoric processes involved.

Methods: Twenty-nine right-handed students took part in the study. Their EEGs were recorded from 128 scalp sites while they viewed gratings of four different spatial frequencies (from 0.75 to 6 $\mathrm{c} / \mathrm{deg}$ ) randomly flashed in the four upper and lower quadrants of the visual field. The tasks consisted of attending and responding to a conjunction of spatial frequency and space location. Intermediate frequencies ( 1.5 and $3 \mathrm{c} / \mathrm{deg}$ ) acted as distracters or lures. Analysis of the ERPs elicited by the same physical stimulus, close in spatial frequency to the actual target and falling within the attended quadrant (pseudo-target) vs. a non-target location, allowed us to identify the time course and neural bases of brain activation during the suppression of irrelevant information.

Results: FAs were on average $9 \%$ for pseudo-targets and $0.2 \%$ for other types of lures, indicating that the former were more difficult to suppress. Target-related ERP components (occipito/ temporal selection negativity, posterior P3b and precentral motor N2) were greater to pseudotargets than other distracters. A large prefrontal negativity $(370-430 \mathrm{~ms})$ was also identified, much larger to pseudo-targets than non-targets (and absent in response to real targets), thus reflecting response inhibition and top-down cognitive control processes.

Conclusion: A LORETA inverse solution identified the neural generators of this effect in the left dorsolateral prefrontal cortex (DLPF), left and right fusiform gyri and bilateral superior temporal cortices. The tentative hypothesis is advanced that these activations might reflect the modulatory effects exerted by the fronto/temporal circuit for the suppression of irrelevant information.
\end{abstract}

\section{Background}

One of the main problems in investigating cognitive or motor suppression processes in go/no-go tasks is that, while go trials are associated with response motor preparation and execution, no-go trials are not, so it is difficult to establish which components are related to response/ 
stimulus suppression typical of no-go trials and which depend on the motor and decision-making processes typical of go trials. Some authors have tried to overcome this problem by comparing motor with count conditions [1-4] or with saccadic eye movements [5] in go trials, or by varying the degree of effort required to withhold the go response [6], or by using a hybrid choice-reaction go/nogo procedure involving selective response priming [7]. The overall pattern of results consists of a fronto-central negative wave (N2) peaking at about $200-400 \mathrm{~ms}$ and an increased frontal P3 response to no-go trials $[2,8,9]$ thought to represent inhibition of responses with no-go stimuli. N2 is sometimes interpreted as a clear sign of response inhibition with a generator in the anterior cingulate cortex [8], and at other times as a non-motoric stage of inhibition, or recognition of the need for inhibition [1]. In other studies, the fronto/central P3 to no-go trials has been associated with response inhibition, generated in the anterior cingulate cortex [10]. Furthermore, the functional significance of N2/P3 effects is debated, since they may represent purely motor inhibition, detection of response conflict, differences in attentional allocation or cognitive inhibition processes.

Notwithstanding the wide literature on this matter, there seems to be no convergence of interpretation. Indeed, Verleger and coauthors [11], who addressed the question whether no-go P3 reflected inhibition of the intended action or resulted from the absence of a negative movement-related potential typical of go trials, advanced the hypothesis that the no-go P3 might reflect monitoring of the withdrawal from overt action, and could be interpreted as the inverse of the negative motor potentials characteristic of go trials. Again, Salisbury and coauthors [4] tried to disentangle the effects of button press on the amplitude of the $\mathrm{P} 3$ component using an auditory go/nogo task in which P300 was measured on button-press and silent-count tasks in control subjects. An estimate of motor activity was constructed from a simple reaction time task, and the motor estimate was subtracted from the button-press P300 according to Kok's formula [9]: true P300 = go P300-motor potentials. The results showed that P300 was smaller and its topography different in the button-pressing task compared to silent-counting, while the motor-correction procedure generated a P300 with normal topography. The authors concluded that no-go P300 responses in button-pressing tasks are confounded by motor potentials, and that motor potential contamination is a real and insidious confounder, which must be dealt with when addressing response inhibition tasks. Overall, the whole issue remains far from understood or resolved because of the intrinsic differences between go and no-go conditions in the oddball paradigm.

The aim of this study was to provide an objective measure of the suppression of non-target stimuli in a conjoined selective visual attention task involving processing of different types of distracters more or less similar to targets, and therefore more or less difficult to suppress. By comparing brain activity relative to pseudo-targets (to which subjects did not actually respond) with that relative to non-targets falling at a non-attended location (easier to suppress), we identified the neural bases of the mechanism by which irrelevant information is suppressed under conditions perfectly comparable from the viewpoint of the motoric processes involved. Gratings of four different spatial frequencies (from 0.75 to $6 \mathrm{c} / \mathrm{deg}$ ) were repeatedly flashed in the four upper and lower quadrants of the left and right visual fields and intermediate frequencies acted as distracters (never being targets in spatial frequency). Analysis of the ERP components elicited by the same physical stimulus, close in spatial frequency to the actual target and falling within the attended vs. unattended location, allowed us to identify the time course and neural bases of brain activation during the suppression of irrelevant information.

\section{Methods}

\section{Participants}

Twenty-nine university students (13 males and 16 females) ranging in age from 20 to 30 years (mean age $=$ 23 years) took part in this experiment as volunteers. All participants had a normal or corrected-to-normal vision with right eye dominance. They were strictly right-handed as assessed by the Edinburgh Inventory and none of them had any left-handed relatives. The experiments were conducted with the understanding and written consent of each participant according to the Declaration of Helsinki (BMJ 1991; 302: 1194) with approval from the Ethical Committee of the Italian National Research Council (CNR) and in compliance with APA ethical standards for the treatment of human volunteers (1992, American Psychological Association). Subjects gained academic credits for their participation. Three subjects were subsequently discarded because of excessive eye-movements.

\section{Stimuli and procedure}

Participants were seated in a dimly lit, electrically shielded cubicle and gazed binocularly on a fixation point permanently present in the centre of a visual display situated $114 \mathrm{~cm}$ in front of them. They were instructed to avoid any kind of eye or body movement. Four square-wave luminance-modulated vertical gratings of $0.75,1.5,3,6 \mathrm{C} /$ deg were randomly presented for $80 \mathrm{~ms}$ in the four quadrants of the visual field. The rectangular patterns were replaced for an interval varying randomly between 690 and $790 \mathrm{~ms}$ (SOA 770-870 ms) with an isoluminant grey field $\left(35 \mathrm{~cd} / \mathrm{m}^{2}\right)$. Stimulus and background had equal average luminance to avoid flash stimulation. The mean grating luminance was measured for each spatial frequency and space location. An ANOVA performed on the luminance values showed no significant difference, thus proving stimulus equiluminance $\left(43 \mathrm{~cd} / \mathrm{m}^{2}\right)$. 
The gratings were randomly presented in pattern-onset mode within the left and right upper and lower hemifields of a PC screen. Within each hemifield, the grating stimulation began $2.5^{\circ}$ above or below the horizontal meridian, and $1.5^{\circ}$ lateral to the vertical meridian, and extended to $3.5^{\circ}$ above or below the horizontal meridian and $5^{\circ}$ along it. Different conjoined selective attention conditions were administered in random order for 0.75 or $6 \mathrm{c} / \mathrm{deg}$ within each hemifield to each subject. Irrespective of target frequency, gratings of 1.5 and $3 \mathrm{c} / \mathrm{deg}$ always served as potential distracters. Before the beginning of each task condition, the participants were instructed to pay conjoined attention to a spatial frequency within a given hemifield (e.g. 6 c/deg in the right upper field) and to ignore the other combinations of frequencies and hemifields. Thus, although the physical stimuli remained unchanged, attention shifted across spatial frequency and space location. While intermediate frequencies falling in an attended quadrant could share the space relevance with actual targets, their frequency relevance depended on their similarity with the latter. In detail, the $3 \mathrm{c} / \mathrm{deg}$ gratings, being 1 octave below $6 \mathrm{c} / \mathrm{deg}$ (an octave change in spatial frequency doubles or halves the frequency), fell close to the 6/deg spatial frequency bandwidth sensitivity, while 1.5 fell close or within the 0.75 spatial frequency bandwidth sensitivity, as demonstrated by psychophysical and VEP studies $[12,13]$. Evidence for a bandwidth of approximately an octave was originally provided by adaptation and masking studies [14,15]. Blakemore and Campbell [14] found that after prolonged observation of a highcontrast sinusoidal grating, gratings of similar spatial frequency were harder to detect; more contrast was needed to see them at threshold. This threshold elevation effect was strongest for test gratings that matched the adapting frequency, and the effect fell to half strength at about $0 \cdot 5$ octave either side of the adapting frequency - hence, a bandwidth of 1 octave. Weak effects were observed with test gratings approaching about 2 octaves below and $1 \cdot 25$ octaves above the adaptation frequency.

For this reason we assumed that frequency relevance might also affect intermediate stimuli, as shown in previous electrophysiological studies [12].

Thus, the same stimulus under different attention conjunction conditions could be: (i) relevant in both spatial location and spatial frequency (pseudo-target), when it fell in the target quadrant and its spatial frequency was 1.5 for $0.75 \mathrm{c} / \mathrm{deg}$ targets or 3 for 6/deg targets; (ii) relevant in spatial location but irrelevant in spatial frequency $(\mathrm{L}+\mathrm{F}-)$, for $1.5 \mathrm{c} / \mathrm{deg}$ gratings when $6 \mathrm{c} / \mathrm{deg}$ was the target and for $3 \mathrm{c} / \mathrm{deg}$ when $0.75 \mathrm{c} / \mathrm{deg}$ was the target; (iii) irrelevant in spatial location but relevant in spatial frequency (L-F+/-); or (iv) irrelevant in both features (L-F-), according to the paradigm devised by Zani and Proverbio [16].
To monitor spatial and stimulus attention selectivity, the volunteers were instructed to press a button in response to targets as accurately and quickly as possible, allowing their reaction times (RT) to be recorded as well. In half the blocks, the participants pushed the detection-RT button with the index finger of the left hand, whereas in the other half they used the right hand. The order of hands was counterbalanced across participants. The order with which the attention tasks were administered and spatial locations attended was counterbalanced across participants and experimental sessions.

\section{EEG recording and analysis}

The EEG was continuously recorded from 128 scalp sites according to the extended international 10-5 system [17] using an elastic cap embedded with tin electrodes. The sampling rate was $512 \mathrm{~Hz}$. Vertical eye movements were recorded by two electrodes placed below and above the right eye, while horizontal movements were recorded from electrodes placed at the outer canthi of the eyes. Linked ears served as the reference lead. The EEG and electro-oculogram (EOG) were amplified with a half-amplitude band pass of 0.016-100 Hz. Electrode impedance was kept below $5 \mathrm{k} \Omega$. EEG epochs were synchronized with the onset of stimulus presentation and analyzed by ANTEEProbe software. Computerized artefact rejection was performed before averaging to discard epochs in which eye movements, blinks, excessive muscle potentials or amplifier blocking occurred. EEG epochs associated with an incorrect behavioural response were also excluded. The artefact rejection criterion was a peak-to-peak amplitude exceeding $50 \mu \mathrm{V}$, and the rejection rate was $\sim 5 \%$. ERPs were averaged offline from $-200 \mathrm{~ms}$ before to $800 \mathrm{~ms}$ after stimulus onset. ERP components were identified and measured with reference to the average baseline voltage over the interval $-100 \mathrm{~ms}$ to $0 \mathrm{~ms}$ relative to stimulus onset.

Low Resolution Electromagnetic Tomography (LORETA [18]) was performed on ERP difference waves at various time latencies. LORETA, which is a discrete linear solution to the inverse EEG problem, corresponds to the 3D distribution of electric neuronal activity that has maximum similarity (i.e. maximum synchronization), in terms of orientation and strength, between neighbouring neuronal populations (represented by adjacent voxels). In this study an improved version of standardized low-resolution brain electromagnetic tomography (sLORETA) was used, which incorporates a singular value decomposition-based lead field weighting: swLORETA [19]. Source space properties were: grid spacing $=10 \mathrm{~mm}$; estimated SNR $=3$.

Distinct ERP averages were obtained for each electrode site, grating spatial frequency, space location, and conjoined-attention condition. Grand-average ERPs were fur- 
ther computed independently of physical stimulus parameters (retinal coordinates and spatial frequency). In this study, only ERPs to intermediate non-target frequencies (i.e. 1.5 and $3 \mathrm{c} / \mathrm{deg}$ ) were analyzed under the various attention conditions, to show the effect of neural suppression of irrelevant stimuli bearing different degrees of similarity to targets. Comparisons were also made with ERPs to effective targets, but an in-depth discussion of attention effects for 0.75 and $6 \mathrm{c} / \mathrm{deg}$ gratings can be found elsewhere [20].

ERP components were quantified by automatically measuring their mean amplitudes across time within the following latency ranges: $230-270 \mathrm{~ms}$ for the occipitotemporal N2 (selection negativity) at P9 and P10 sites, 275$315 \mathrm{~ms}$ for the N2 motor potential at FCC1h and FCC2h sites, 370-430 ms for the prefrontal NP400 component at PF1 and PF2 sites, and 380-500 ms for the posterior P3b component at PPO1 and PPO2 sites.

Separate two-way repeated-measure analyses of variance (ANOVAs) were performed on the mean values computed for each individual subject as a function of the attention condition, and independent of physical stimulus parameters. Factors were: attentional relevance $(\mathrm{L}+\mathrm{F}+/-, \mathrm{L}+\mathrm{F}-, \mathrm{L}-$
F+/-, L-F-) and cerebral hemisphere (right and left). Possible type 1 errors associated with inhomogeneity of variance were controlled by the Greenhouse-Geisser procedure. Post-hoc Tukey tests were used for multiple comparisons of means.

\section{Results}

The FA rate was extremely low and ranged from $0.2 \%$ to all types of non-targets (including those of the target frequency: 0.75 and $6 \mathrm{c} / \mathrm{deg}$ ) to an average of $8.77 \%$ for pseudo-targets falling at the attended location $(\mathrm{L}+)$ and within the target spatial frequency bandwidth $(\mathrm{F}+/-)$, as illustrated in Figure 1 (6.63\% for the attend- 0.75 condition, and $10.90 \%$ for the attend- 6 condition). The fact that gratings falling at the attended location and within the target's spatial frequency bandwidth (pseudo-targets) elicited 40 times more FAs than other types of lures indicates how similar they were to targets and how difficult they were to suppress at both the cognitive and response preparation levels.

Figure 2 shows ERP waveforms recorded over posterior scalp sites in response to lure gratings of 1.5 and $3 \mathrm{c} / \mathrm{deg}$, sharing or not sharing space location with the target (L+ or L-) and falling or not falling within the same spatial fre-

\section{FAs distribution (\%)}

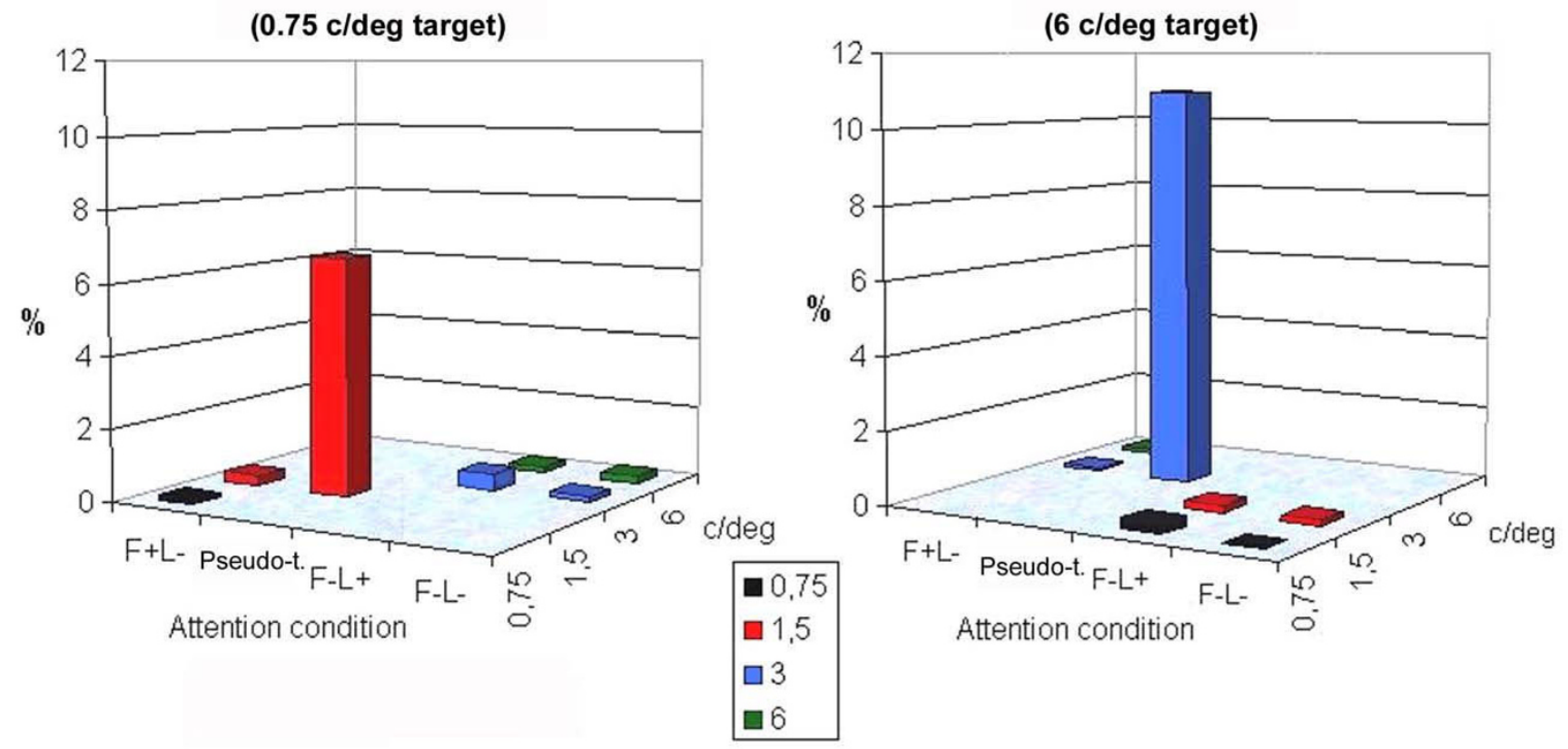

Figure I

False alarm distribution (percentages \%) as a function of attention condition and grating spatial frequency. It is evident that gratings falling at the attended location and within the target's spatial frequency bandwidth received the most of the FAs and were therefore considered as pseudo-targets. 
quency bandwidth as the target. Strong frequency-based attentional effects are visible for both types of grating (especially at the selection negativity level), suggesting that when attention was paid to $0.75 \mathrm{c} / \mathrm{deg}$ the most similar gratings were $1.5 \mathrm{c} / \mathrm{deg}$, and when attention was paid to 6 $\mathrm{c} / \mathrm{deg}$ the most similar gratings were $3 \mathrm{c} / \mathrm{deg}$ in frequency.

In fact, ERP analysis revealed strong attentional effects for intermediate frequencies (lure stimuli) presented at the attended location and falling within the same spatial fre-

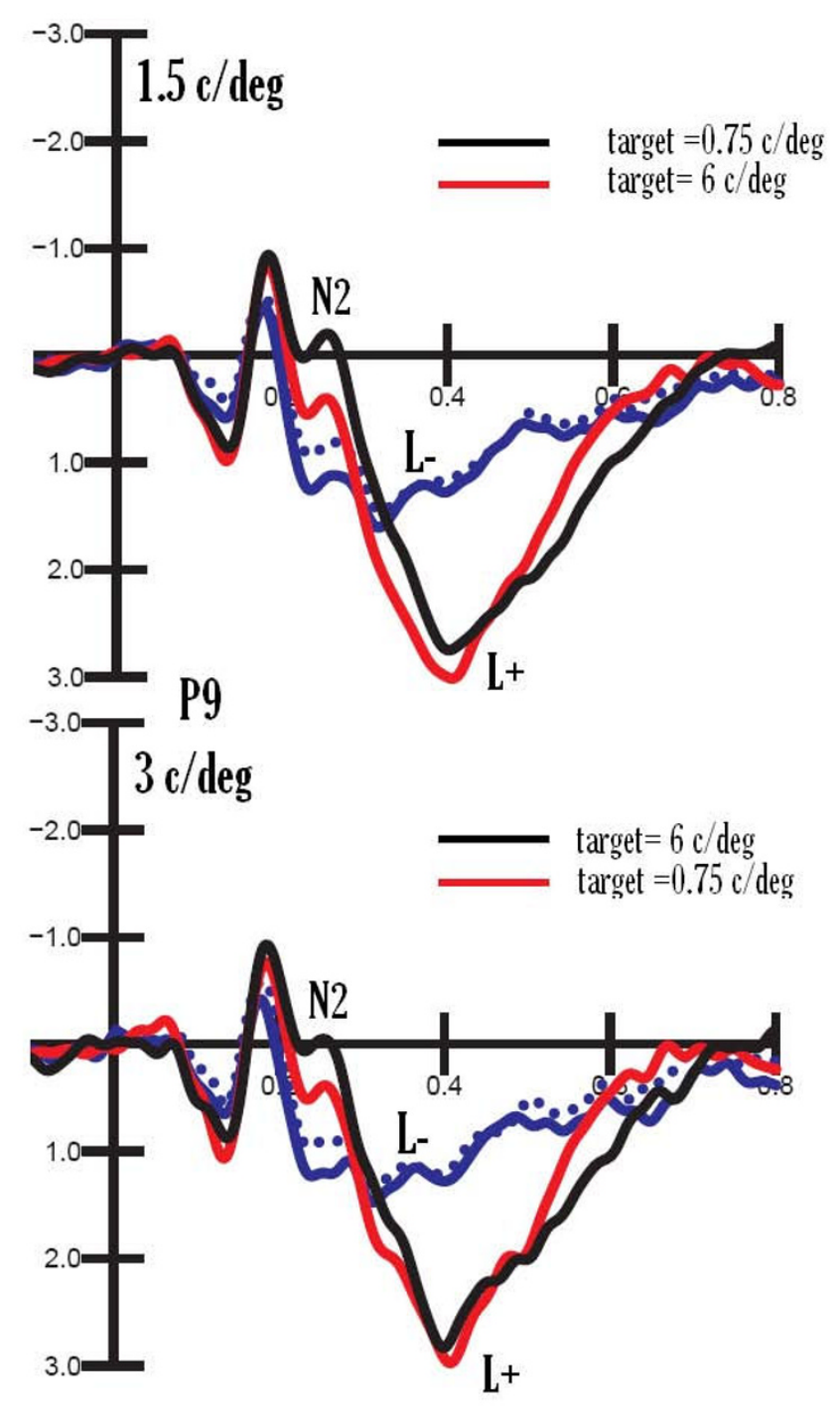

Figure 2

Grand-average ( $N=26)$ ERP waveforms recorded over the left occipito-temporal region in response to gratings of 1.5 and $3 \mathrm{c} / \mathrm{deg}$, sharing or not sharing space location with the target ( $L+$ or $L-)$ and falling or not falling within the same spatial frequency bandwidth as the target. quency bandwidth as actual targets (see Figure 3). They included typical attentional selection negativity over the occipito/temporal area (N2), a posterior P3 component, and a motor precentral N2 component larger to pseudotargets than L+F- stimuli, and to the former categories than to location-irrelevant gratings. Even earlier C1 and P1 spatial frequency and location-relevant effects are visible from the grand-average ERPs, further supporting the evidence for early spatial frequency-based selective attention effects for target stimuli [20].

Statistical analyses performed on the mean area amplitude of posterior N2 (attentional selection negativity) showed that the attention condition was highly significant $(\mathrm{F}[3,75]=16.06$, GG adjusted $\mathrm{p}<0.000059)$ with larger negativities to pseudo-targets than stimuli sharing only space location with the targets, and to the latter than to non-targets (see mean amplitude values in Figure 4). The attention condition was also strongly significant for the P3b posterior component $(\mathrm{F}[3,75]=23.35$, GG adjusted $\mathrm{p}<0.00002$ ), with larger positivities to pseudotargets than stimuli sharing only space location with the targets, and to the latter than to other lures. P3b to pseudo-targets was of greater amplitude over the left than the right hemisphere, as indicated by the significant hemisphere $\times$ attention interaction and relative post-hoc comparisons $(\mathrm{F}[3,75]=10.97 \mathrm{GG}$ adjusted $\mathrm{p}<0.000021)$. At precentral sites, the motor $\mathrm{N} 2$ potential was also affected by the attention condition ( $\mathrm{F}[3,75]=8.61$, GG adjusted, $\mathrm{p}<0.0026)$, with larger negativities to pseudo-targets than to all other distracters. Similarly, the prefrontal NP400 was strongly affected by the attention condition (F $[3,75]=6.48$, GG adjusted, $\mathrm{p}<0.00087)$, with larger negativities to pseudo-targets than to all other stimulus categories. Since mean reaction times occurred at about 500 ms of latency (mean RT $=510 \mathrm{~ms}$ ) it was hypothesized that the NP400 prefrontal response (370-430 ms), the only potential markedly larger to the most-similar-as-possible-to-targets distracters (pseudo-targets), which were more difficult to suppress, might be considered a sign of neural suppression. Indeed, pseudo-targets showed all signs of being processed as targets except for this last sign of non-targetness (N3) followed by a lack of motor response.

In order to investigate the neural bases of the suppression effect for pseudo-targets, a difference wave was computed by subtracting the ERPs to lures that were less difficult to suppress (frequency-pseudorelevant $(\mathrm{F}+)$ but locationirrelevant (L-)) from the ERPs to pseudo-targets (locationrelevant and falling within the relevant spatial frequency bandwidth). A LORETA inverse solution was therefore performed on the difference wave in the time window $370-430 \mathrm{~ms}$. The neural generators explaining the surface difference voltage are shown in Figure 5 and their Taila- 


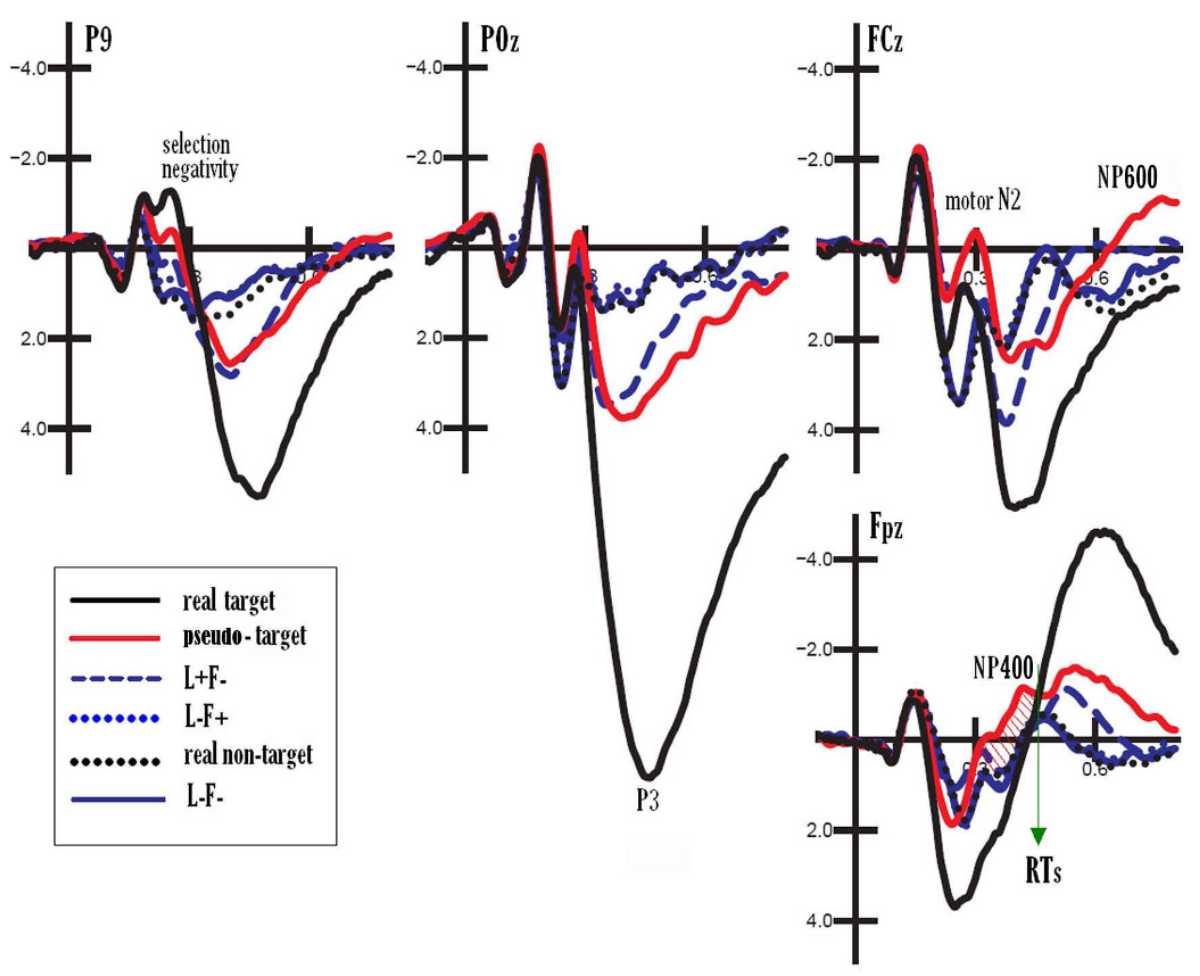

Figure 3

Grand-average ( $N=26)$ ERP waveforms recorded at left occipito/temporal (P9), midline occipital/parietal (POz), precentral (FCz) and prefrontal (PFz) sites as a function of attention condition and independent of stimulus spatial frequency. ERPs elicited by real targets (the extreme frequencies 0.75 and $6 \mathrm{c} / \mathrm{deg}$ ) are in black, whereas the ERPs elicited by lures are in colour. The only component elicited by pseudo-targets that did not clearly indicate targetness was the prefrontal N3 deflection.

rach coordinates are listed in Table 1 . The active sources included the left prefrontal cortex (BA9), the left and right superior temporal gyrus (BA38), and the left and right fusiform gyrus of the temporal cortex (BA19/20), with a left hemispheric asymmetry in the magnitude of activation (nA).

\section{Discussion}

The aim of the study was to investigate the neural bases of executive control mechanisms involved in the ability to suppress irrelevant visual information and inappropriate motor responses. ERPs to intermediate irrelevant spatial frequencies were examined in the context of a conjoined space- and frequency-based selective attention task. The analysis of false alarm rates proved that non-target stimuli falling at the attended location $(\mathrm{L}+)$ and within the target spatial frequency bandwidth (pseudo-targets) were more difficult to suppress in that they elicited an average of $8.77 \%$ of FAs. Pseudo-target responses were characterized by a pronounced occipito/temporal selection negativity [16,21-23] indicating perceptual similarity to target gratings. At posterior sites, pseudo-targets elicited a large P3b component probably reflecting voluntary allocation of visual attention to targets as described as a key function of the parietal cortex by Cabeza and coworkers [24]. Consistent with this pattern of results, a marked N2 peak was visible at fronto-central sites, very probably indicating motor preparation processes $[9,25]$; it was of greater amplitude to pseudo-targets than to other distracters, which exhibited a sort of frontal P3 response instead. In this context, the frontal P3 cannot be interpreted as a sign of suppression, as in many go-no/go paradigms $[1,10,26]$, since it was much lower in response to lures that were most difficult to suppress (i.e. pseudo-targets), as demonstrated by the false alarm distributions. Therefore, the present data do not support the view $[2,8,9]$ that frontal P3 response to no-go trials might represent inhibition of responses with no-go stimuli. On the other hand, P3 might be conceptualized as a lack of motor preparation and of the negative voltage response execution processes typical of go trials $[4,11]$, which were found in association with pseudo-target presentation, because of their striking similarity to real targets. In addition, it might also indicate a sort of P3b reflecting monitoring processing and stimulus evaluation. Furthermore, since no response was emitted to any of the lures considered in this study (since ERPs associated with 

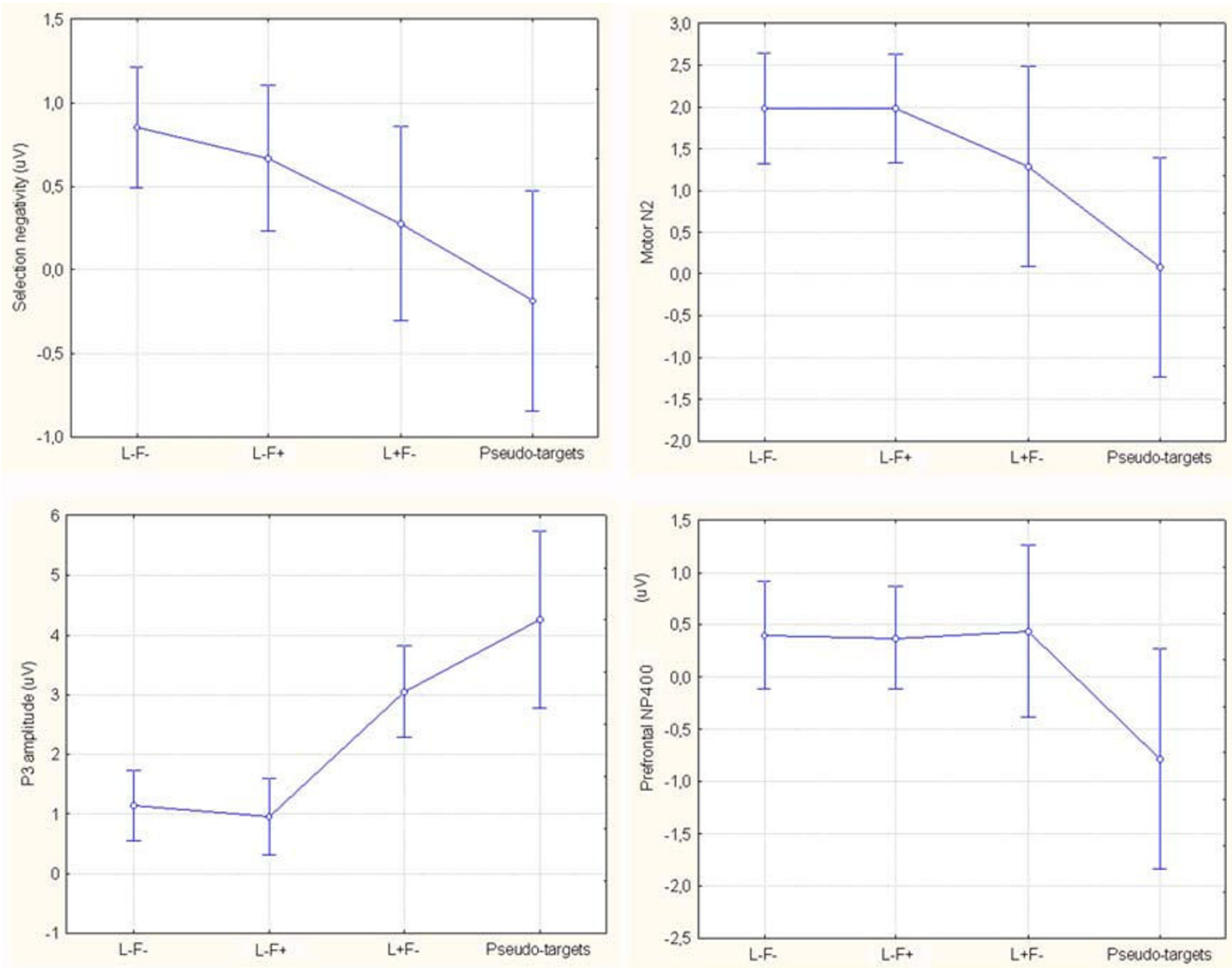

Figure 4

Mean amplitude values (in $\mu \mathrm{V}$ ) of ERP components of interest recorded as a function of attention condition and independent of grating spatial frequency. Error bars reflect standard errors.

incorrect trials were rejected), pseudo-targets and other distracters are perfectly comparable because they represent the brain processing of the same physical stimulus under different attention conditions, and the ERPs are not contaminated by overt motor responses as in go/no-go paradigms.

Inspection of the waveforms of Figure 3 reveals that motor N2 appeared larger to pseudo-targets than to targets, and one might be tempted to identify this component as the $\mathrm{N} 2$ response described in the oddball literature as being greater to no-go than go trials (e.g. [8]. However, this differential effect might very well be because the overlapping positivity (typical of space-relevant $\mathrm{L}+$ stimuli) of pseudotargets is lower than to real targets. Unfortunately, there- fore, it cannot be demonstrated that frontal N2 to non-targets was a sign of motor suppression in this specific case, although the possibility cannot be excluded a priori.

At prefrontal sites a negative deflection (NP400) was identified in the $370-430 \mathrm{~ms}$ time window, which was not characteristic of target stimuli (as proved by a direct comparison with the ERPs elicited by real targets at Fpz, in Figure 3) and was much smaller in response to lures falling at an unattended location or outside the target spatial frequency bandwidth. For this reason, we hypothesized that the NP400 deflection might reflect the brain activity linked to the suppression of irrelevant information and/or the inhibition of inappropriate responses. The present study does not address the question of whether NP400 


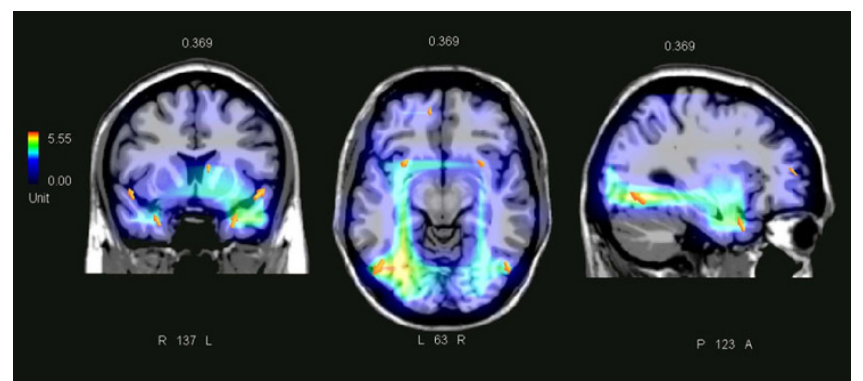

Figure 5

swLORETA [19] inverse solution displaying the neural generators of the $\mathrm{N} 3$ suppression effect for pseudo-targets. LORETA was computed on the difference wave obtained by subtracting ERPs to L-F+ from ERP to pseudo-targets in the time window $370-430 \mathrm{~ms}$, corresponding to the maximum amplitude of the prefrontal N3 response. A realistic boundary element model (BEM) was derived from a TI weighted 3D MRI data set by segmentation of the brain tissue. The BEM model consisted of one homogenic compartment made up of 3446 vertices and 6888 triangles. The head model was used for intra-cranial localization of surface potentials. Segmentation and head model generation were performed using the ASA (A.N.T. Software B.V., Enschede, the Netherlands) package [45]. The electromagnetic dipoles are shown as arrows and indicate the position, orientation and magnitude of dipole modelling solution applied to the ERP difference wave in the specific time window $(370-430 \mathrm{~ms})$. The different colours represent differences in the magnitude of the electromagnetic signal (in $\mathrm{nA}$ ). 0.369 indicates the boundary of time window $(370 \mathrm{~ms}$ at $5 / 2$ $\mathrm{Hz}$ of sampling rate). $\mathrm{L}=$ left; $\mathrm{R}=$ right; $\mathrm{P}=$ posterior; $\mathrm{A}=$ anterior; numbers refer to the displayed brain slice in coronal, axial and sagittal views, respectively.

might indicate a suppression of motor or cognitive information, or conflict monitoring vs. response inhibition, but focuses on the finding of a clear sign that irrelevant information is suppressed without the problems inherent to the go/no-go paradigm.
In summary, notwithstanding the apparent similarity between motor N2 and NP400, the two components, one peaking at about $300 \mathrm{~ms}$ over precentral sites, the other at about $430 \mathrm{~ms}$ at prefrontal sites, were quite different in nature, the former (motor N2) being very pronounced in response to real targets and indicating targetness, and the latter being very pronounced in response to lures and indicating non-targetness). They also differed in terms of scalp distribution, as visible in topographical maps of Figure 6, displaying the voltage distribution of motor N2, prefrontal NP400 and frontal NP600 at anterior electrode sites.

The enhanced NP400 to pseudo-targets than other lures less difficult to suppress persisted at frontal sites in the form of a large negative NP600 deflection. The evidence that this potential was still larger to pseudo-targets than other lures at both $\mathrm{Fpz}$ and $\mathrm{FCz}$, and even larger to the former stimuli than real targets at FCz sites (see Figure 3) suggests a possible functional similarity with NP400, and its role in the sustained suppression of irrelevant visual information. The problem with NP600, however, is that it was larger in amplitude to targets than pseudo-targets at prefrontal sites, rendering it difficult to fully understand its functional meaning. Proponents of the no-go related frontal P3 might hypothesize that P400 (the wide-spread positivity visible in the left upper map of Figure 7), and not only prefrontal N400, is indeed a reflection of cortical inhibition of irrelevant stimuli. However, this hypothesis is countered by the evidence that P400 was much larger to target than pseudo-targets, therefore indexing stimulus selection rather than inhibition. Thus, the question remains unsolved.

It is interesting to note that the onset of prefrontal NP400, in our study, followed the stage of motor preparation indicated by precentral N2, and preceded the latency of response times (indicated by the green arrow in Figure 3) that corresponded to an average of $510 \mathrm{~ms}$.

Table I: Tailarach coordinates corresponding to the intracranial generators explaining the difference voltages related to the pseudotarget suppression effect in the 370-430 ms time window, according to swLORETA [18]; grid spacing = $10 \mathrm{~mm}$, estimated SNR = 3 .

\begin{tabular}{cccccccc}
\hline Magnit. & T-x $[\mathbf{m m}]$ & T-y $[\mathbf{m m}]$ & T-z $[\mathbf{m m}]$ & H & Lobe & Area \\
\hline 2.492 & 50.8 & 23.6 & -22.9 & RH & Temporal & Fusiform gyrus \\
\hline 3.726 & 31.0 & 8.2 & -20.0 & RH & Temporal & Superior Temp. gyrus \\
\hline 4.402 & 28.5 & 8.2 & -20.0 & LH & Temporal & Superior Temp. gyrus \\
\hline 4.165 & 50.8 & -66.1 & -10.5 & RH & Temporal & Fusiform gyrus \\
\hline 5.549 & -48.5 & -66.1 & -10.9 & LH & Temporal & Fusiform gyrus \\
\hline 3.035 & -8.5 & 44.4 & 15 & LH & Frontal & Medial frontal gyrus
\end{tabular}




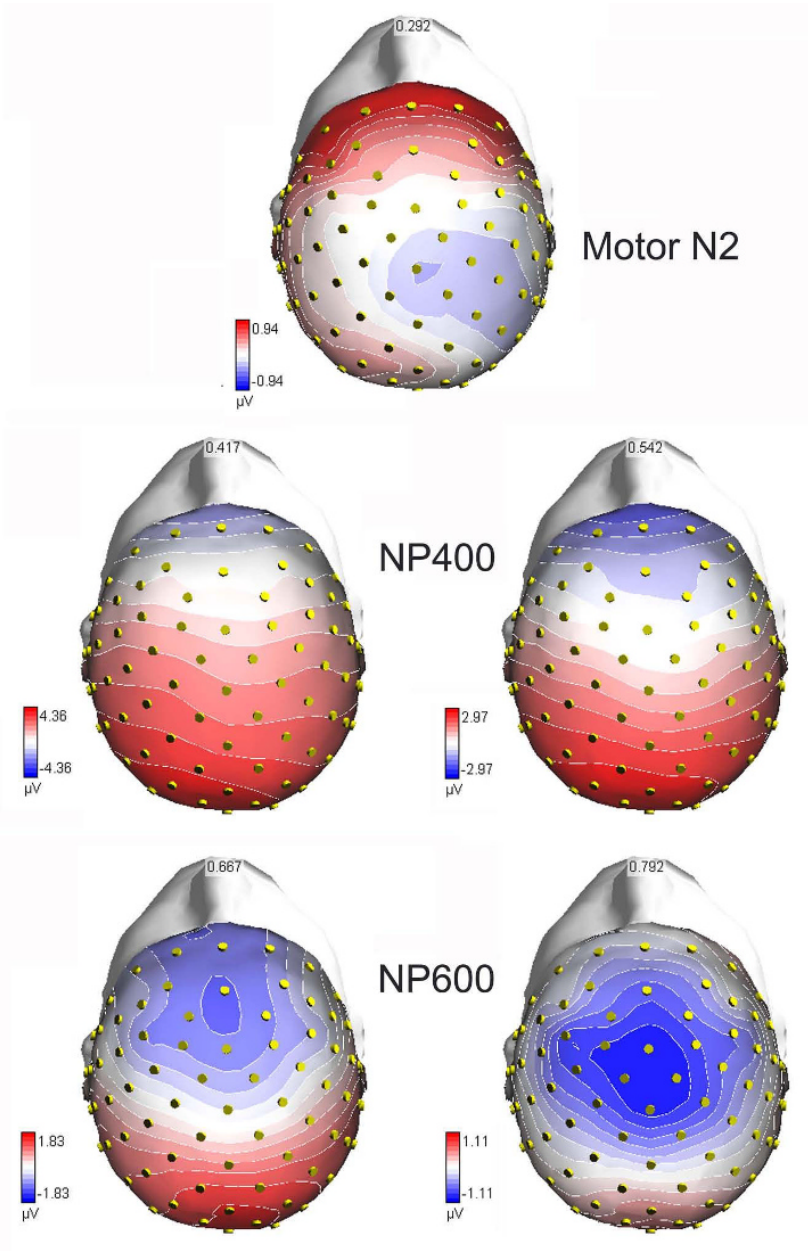

Figure 6

Time series of voltage topographical maps (anteriortop view) relative to pseudo-target processing and computed every I 25 from 292 ms to 792 ms. Maps were made by plotting colour-coded isopotentials derived by interpolating voltage values between scalp electrodes at specific latencies.

In another study, a negative event-related brain potential deflection (N470) was described, the generator of which was located in the anterior cingulate cortex; it was possibly related to response inhibition in a delayed response task [27] and was similar in morphology to the N430 described in the present conjoined selective visual attention task. However, while the anterior cingulate cortex seems most involved in the conflict monitoring $[28,29]$ typical of go/no-go tasks, the prefrontal cortex seems more involved in the top-down modulation of attentional processes typical of conjoined selective attention tasks. Several neuroimaging and neurophysiological studies have provided evidence for a role of the dorsolateral prefrontal cortex (DLPF:BA9/46) in the suppression of motor behaviour [30-32] and in cognitive control [33,34].
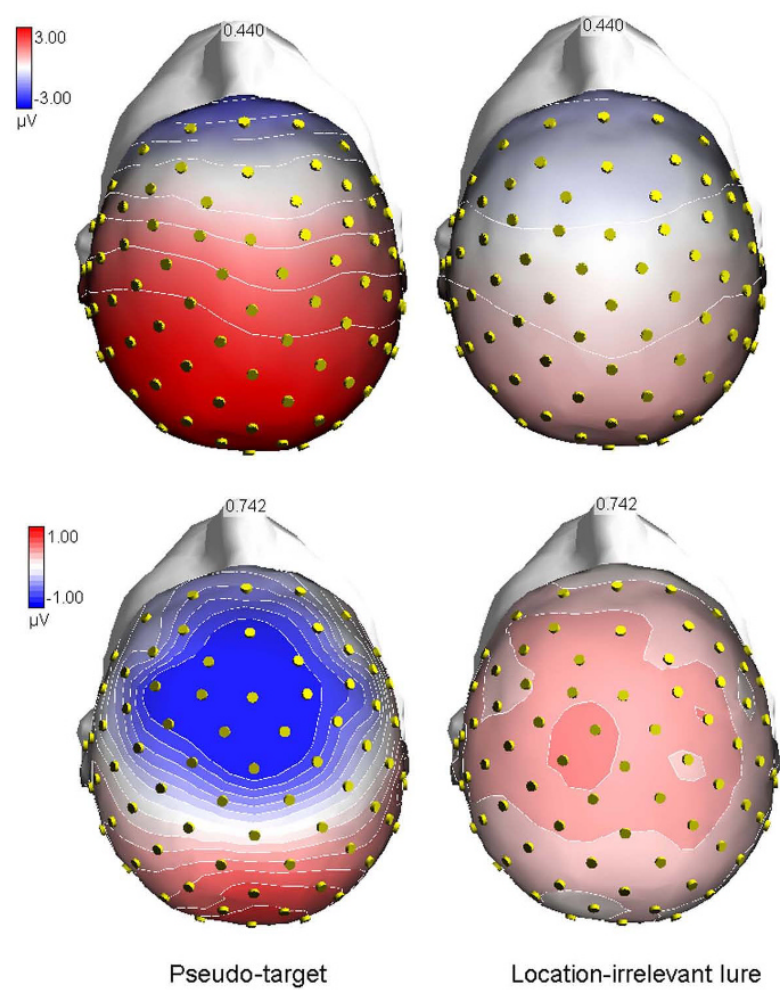

Figure 7

Voltage topographical maps (anterior-top view) relative to pseudo-targets (left column) and L-F+ gratings (right column) processing. As explained in the text, while prefrontal $\mathrm{N} 400$ very likely reflected cortical inhibition of lures difficult to suppress, the widespread positivity, which was clearly absent to non-targets, was probably a sign of (reduced) targetness of gratings falling in the same location and spatial frequency bandwidth as targets.

Activation of the dorsolateral prefrontal cortex, during both inhibition of the prepotent impulse to respond and the suppression of irrelevant stimuli, is consistent with the view that this region is involved in cognitive control processes. Previous studies have reported a linear relationship between DLPFC activation and the degree of cognitive control [29,35]. Consistent with these data, Blasi and coauthors [31] found greater DLPFC activation during the relatively more difficult no/go condition (as reflected by performance scores that were poorer than under other conditions) than during the go condition.

It is also known that patients with prefrontal lesions confined to BA areas 9 and 46 are impaired in their ability not only to focus attention on task-relevant stimuli [36] but also to suppress task-irrelevant information [37]. In our study, the bilaterally increased occipito/temporal and superior temporal activation found in the LORETA inverse solution for the N3 suppression effect suggests a marked 
attentional modulation of the ventral stream, probably reflecting the activity of the ipsilateral fronto-temporal circuit described by Knight and other authors [38-40]. Indeed, it has been shown that the prefrontal cortex exerts modality-specific GABA-mediated suppression of sensory transmission through thalamic relay nuclei. The prefrontal cortex also has a role in response inhibition (the cognitive process required to cancel an intended movement), that is, in the suppression of inappropriate responses. In fact, it has been shown that the inferior frontal cortex is able to suppress an already-initiated manual response through a direct fronto-striatal pathway involving the subthalamic nucleus of the basal ganglia [41].

Our study revealed indications of hemispheric asymmetry in the modulatory attention effects: a greater posterior P3b component was found to pseudo-targets over the left than the right hemisphere. Again, swLORETA showed a left-sided activation of the left dorsolateral prefrontal cortex and an enhanced activation for pseudo-targets that was greater in the left than the right fusiform gyrus. Overall, these findings might suggest that the left hemisphere has better selective capability when dealing with gratings of similar spatial frequencies, so it requires a narrower attentional focus [42-44].

\section{Limitations}

A possible limitation of the study, as suggested by one of our referees, is that the electrode material (pure tin) that is commonly used for EEG caps (Electro-Cap International, Inc.) might have induced a polarization for which late NP600 and, possibly, NP400 might represent the overshooting high-pass filtered P3b due to high impedance for slow oscillations. However we regard this suspicion as highly hypothetical.

\section{Conclusion}

In summary, the combined observation of false alarms (FAs) and event-related potentials to distracter stimuli in a selective attention task to a conjunction of space location and spatial frequency of gratings showed that irrelevant stimuli that are more difficult to suppress (pseudotargets) featured the ERP components typical of targets: occipito/temporal selection negativity, posterior P3b and precentral motor N2. In addition, they exhibited a large negativity at the prefrontal area $(370-430 \mathrm{~ms})$, following the motor preparation stage (275-315 ms) and preceding the reaction time stage (about $510 \mathrm{~ms}$ ), which was the best candidate for reflecting response inhibition and topdown cognitive control. The swLORETA inverse solution $[19,45]$ identified the neural generators of this effect in the left dorsolateral prefrontal cortex (BA9), left and right fusiform gyri (with left hemispheric asymmetry) and bilateral superior temporal cortices. We advance the hypothesis that these activations might reflect the modulatory effects exerted by the fronto/temporal circuit for the suppression of irrelevant information. However, further investigation will be certainly needed to corroborate any interpretation.

\section{Competing interests}

The authors declare that they have no competing interests.

\section{Authors' contributions}

AMP conceived of and coordinated the study, interpreted the data and drafted the manuscript. MDZ acquired, processed, and analyzed the ERP data, NC analyzed behavioural data, AZ was involved in the design of the paradigm, interpretation of data, and revision of the manuscript. All authors read and approved the final manuscript.

\section{Acknowledgements}

The authors are grateful to Roberta Adorni, Valentina Rossi and Friederike Wiedemann for their help with EEG recording. They also wish to thank Monte Tabor Foundation of HSR Hospital for providing a research fellowship to MDZ.

\section{References}

I. Smith JL, Johnstone SJ, Barry RJ: Movement-related potentials in the Go/NoGo task: The $\mathbf{P} 3$ reflects both cognitive and motor inhibition. Clin Neurophysiol 2008, I I 9:704.

2. Pfefferbaum A, Ford JM, Weller BJ, Kopell BS: ERPs to response production and inhibition. Electroencephalogr Clin Neurophysiol 1985, 60:423-434.

3. Bruin $\mathrm{KJ}$, Wijers $\mathrm{AA}$ : Inhibition, response mode, and stimulus probability: a comparative event-related potential study. Clin Neurophysiol 2002, I I 3: I I72.

4. Salisbury DF, Rutherford B, Shenton ME, McCarley RW: Buttonpressing affects P300 amplitude and scalp topography. Clin Neurophysiol 200I, I I 2: I676

5. Van't Ent D, Apkarian P: Motoric response inhibition in finger movement and saccadic eye movement: a comparative study. Clin Neurophysiol 1999, I 1 0:1058.

6. Jodo E, Kayama Y: Relation of a negative ERP component to response inhibition in a Go/No-go task. Electroencephalogr Clin Neurophysiol 1992, 82:477.

7. Kopp B, Mattler U, Goertz R, Rist F: N2, P3 and the lateralized readiness potential in a nogo task involving selective response priming. Electroencephalogr Clin Neurophysiol 1996, 99: 19.

8. Bekker EM, Kenemans JL, Verbaten MN: Source analysis of the N2 in a cued Go/NoGo task. Brain Res Cogn Brain Res 2005, 22:22I.

9. Kok A: Overlap between P300 and movement-related-potentials: A response to Verleger. Biol Psychol I988, 27:5I.

10. Beste C, Saft C, Andrich J, Gold R, Falkenstein M: Response inhibition in Huntington's disease - A study using ERPs and sLORETA. Neuropsychologia 2008, 46: I 290.

II. Verleger R, Paehge T, Kolev V, Yordanova J, Jaskowski P: On the relation of movement-related potentials to the go/no-go effect on P3. Biol Psychol 2006, 73:298.

12. Zani A, Proverbio AM: ERP signs of early selective attention effects to check size. Electroencephalogr Clin Neurophysiol 1995, 95:277-292

13. DeValois RL, Albrecht DG, Thorel LG: Spatial frequency selectivity of cells in the macaque visual cortex. Vision Res 1982 , 22:545-559.

14. Blakemore C, Campbell FW: On the existence of neurones in the human visual system selectively sensitive to the orientation and size of retinal images. J Physiol. 1969, 203(I):237-260.

15. Pantle A, Sekuler R: Size-detecting mechanisms in human vision. Science 1968, 162:||46-1|48.

16. Zani A, Proverbio AM: Attention modulation of short latency ERPs by selective attention to conjunction of spatial frequency and location. J Psychophysiol 1997, I I:2 I-32. 
17. Oostenveld R, Praamstra P: The five percent electrode system for high resolution EEG and ERP measurements. Clin Neurophysiol 2001, I I 2:713-719.

18. Pasqual-Marqui RD, Michel CM, Lehmann D: Low resolution electromagnetic tomography: a new method for localizing electrical activity in the brain. Int J Psychophysiol I 994, I 8:49-65.

19. Palmero-Soler E, Dolan K, Hadamschek V, Tass PA: swLORETA: a novel approach to robust source localization and synchronization tomography. Phys Med Biol 2007, 52:I783-I800.

20. Proverbio AM, Del Zotto $M$, Zani A: Electrical neuroimaging evidence that spatial frequency-based selective attention affects VI activity as early as $\mathbf{4 0} \mathbf{~ m s}$ in humans. in press.

21. Zani A, Proverbio AM: ERP signs of frontal and occipital processing of visual targets and distracters within and without the channel of spatial attention. Focus on Neuropsychology Research 2006:38-88.

22. Previc FH, Harter MR: Electrophysiological and behavioral indicants of selective attention to multifeature gratings. Percept Psychophys 1982, 32:465-472.

23. Harter MR, Previc FH: Size-specific information channels and selective attention: visual evoked potential and behavioral measures. Electroencephalogr Clin Neurophysiol 1978, 45:628-640.

24. Cabeza R, Ciaramelli E, Olson IR, Moscovitch M: The parietal cortex and episodic memory: an attentional account. Nat Rev Neurosci 2008, 9:613.

25. Starr A, Sandroni $P$, Michalewski HJ: Readiness to respond in a target detection task: pre- and post-stimulus event-related potentials in normal subjects. Electroencephalogr Clin Neurophysiol 1995, 96:76.

26. Strik WK, Fallgatter AJ, Brandeis D, Pascual-Marqui RD: Threedimensional tomography of event-related potentials during response inhibition: evidence for phasic frontal lobe activation. Electroencephalogr Clin Neurophysiol 1998, I 08:406.

27. Qiu J, Li H, Liu Q, Zhang Q: Brain mechanism of response execution and inhibition: an event-related potential study. Neuroreport. 2008, I 9(I): I2I-I25.

28. Haupt S, Axmacher N, Cohen MX, Elger CE, Fell J: Activation of the caudal anterior cingulate cortex due to task-related interference in an auditory Stroop paradigm. Hum Brain Mapp 2009 in press.

29. MacDonald AW, Cohen JD, Stenger VA, Carter CS: Dissociating the role of the dorsolateral prefrontal and anterior cingulate cortex in cognitive control. Science 2000, 288: |835-1838.

30. Daskalakis ZJ, Farzan F, Barr MS, Maller JJ, Chen R, Fitzgerald PB: Long-interval cortical inhibition from the dorsolateral prefrontal cortex: a TMS-EEG study. Neuropsychopharmacology 2008, 33:2860.

31. Blasi G, Goldberg TE, Weickert T, Das S, Kohn P, Zoltick B, Bertolino $A$, Callicott JH, Weinberger DR, Mattay VS: Brain regions underlying response inhibition and interference monitoring and suppression. Eur J Neurosci 2006, 23:1658-I664.

32. Nyffeler T, Müri RM, Bucher-Ottiger Y, Pierrot-Deseilligny C, Gaymard B, Rivaud-Pechoux S: Inhibitory control of the human dorsolateral prefrontal cortex during the anti-saccade paradigm; a transcranial magnetic stimulation study. Eur J Neurosci 2007, 26: | 38|- I 385.

33. Desimone R, Duncan J: Neural mechanisms of selective visual attention. Annu Rev Neurosci 1995, 18:193-222.

34. Miller EK, Cohen JD: An integrative theory of prefrontal cortex function. Annu Rev Neurosci 2001:167-202.

35. Kerns JG, Cohen JD, MacDonald AW III, Cho RY, Stenger VA, Carter $\mathrm{CS}$ : Anterior cingulate conflict monitoring and adjustments in control. Science 2004, 303:1023-1026.

36. Chao LL, Knight RT: Contribution of human prefrontal cortex to delay performance. J Cogn Neurosci 1998, I0:167-I77.

37. Chao LL, Knight RT: Human prefrontal lesions increase distractibility to irrelevant sensory inputs. Neuroreport. 1995, 2 I(12): I605-1610.

38. Wang L, Liu X, Guise KG, Knight RT, Ghajar J, Fan J: Effective connectivity of the fronto-parietal network during attentional control. J Cogn Neurosci 2009 in press.

39. Barceló F, Suwazono S, Knight RT: Prefrontal modulation of visual processing in humans. Nat Neurosci 2000, 3:399-403.

40. Bradley RP: Delay-period activity in the prefrontal cortex: one function is sensory gating. J Cogn Neurosci 2005, I 7:1679-1690.
4I. Aron AR, Poldrack RA: Cortical and subcortical contributions to stop signal response inhibition: role of the subthalamic nucleus. J Neurosci 2006, 26:2424-2433.

42. Proverbio AM, Zani A, Avella C: Hemispheric asymmetries for spatial frequency discrimination in a selective attention task. Brain Cogn 1997, 34:3II.

43. Proverbio AM, Minniti A, Zani A: Electrophysiological evidence of a perceptual precedence of global vs. local visual information. Brain Res Cogn Brain Res 1998, 6:321.

44. Yamaguchi S, Yamagata S, Kobayashi S: Cerebral asymmetry of the "top-down" allocation of attention to global and local features. J Neurosci. 2000, 20(9):RC72.

45. Zanow F, Knösche TR: ASA-Advanced Source Analysis of Continuous and Event-Related EEG/MEG Signals. Brain Topogr 2004, 16:287.
Publish with Bio Med Central and every scientist can read your work free of charge

"BioMed Central will be the most significant development for disseminating the results of biomedical research in our lifetime. "

Sir Paul Nurse, Cancer Research UK

Your research papers will be:

- available free of charge to the entire biomedical community

- peer reviewed and published immediately upon acceptance

- cited in PubMed and archived on PubMed Central

- yours - you keep the copyright 